\title{
Diffusion anisotropy and void development under cascade irradiation
}

\author{
A.A. Semenov • C.H. Woo $\cdot$ W. Frank
}

Received: 8 July 2008 / Accepted: 11 July 2008 / Published online: 29 July 2008

(C) The Author(s) 2008. This article is published with open access at Springerlink.com

\begin{abstract}
Cascade irradiation of metals gives rise to swelling as a result of the creation of voids and the evolution of the void ensemble. Under suitable circumstances, the originally disordered void distribution transforms into to a void lattice. As demonstrated previously, the understanding of the evolution and the unique features of the void ensemble requires a difference in the anisotropy of the diffusion (DAD) of vacancies and self-interstitial atoms (SIAs), which is achieved by one-dimensional diffusion of the SIAs. On the other hand, void swelling has been successfully modeled in terms of three-dimensional diffusion of both vacancies and SIAs. In the present paper it is shown that these seemingly contradicting interpretations and all related observations can be quantitatively reconciled by a small DAD created by only $\sim 1 \%$ of SIAs diffusing one-dimensionally. It is also demonstrated that at the initial stage of void-lattice formation, ordering occurs mainly on close-packed crystal planes, which is in contrast to the naïve expectation that one-
\end{abstract}

The paper is dedicated to Professor Dr. Dr. h. c. Alfred Seeger on the occasion of his 80th birthday.

A.A. Semenov · C.H. Woo $(\varangle)$

Department of Electronic and Information Engineering, The Hong

Kong Polytechnic University, Kowloon, Hong Kong SAR, China

e-mail: chung.woo@polyu.edu.hk

\section{A.A. Semenov}

Institute for Nuclear Research, Russian Academy of Sciences,

Moscow, Russia

W. Frank

Max-Planck-Institut für Metallforschung, Stuttgart, Germany

W. Frank

Institut für Theoretische und Angewandte Physik, Universität

Stuttgart, Stuttgart, Germany dimensional diffusion of SIAs should result in a void ordering along close-packed directions. Finally it is found that, in the case of a small DAD, voids annihilate via stochastic shrinkage much faster than by coalescence. This falsifies the argument in the literature that one-dimensional diffusion of SIAs would necessarily lead to the coalescence of voids and destabilization of the void lattice.

PACS $61.80 . \mathrm{Az} \cdot 61.80 . \mathrm{Hg} \cdot 61.72 . \mathrm{Cc}$

A difference between the anisotropies of diffusion of vacancies and self-interstitials (henceforth called Diffusional Anisotropy Difference or, briefly, DAD) gives rise to a bias that plays an important role in the irradiation-induced evolution of microstructures in metals. Based on Gösele's work [1] on reaction kinetics, the DAD effect was first introduced by Woo and Gösele [2] to explain irradiation growth. Later irradiation creep and void-lattice formation were interpreted in terms of DAD by Woo [3] and Woo and Frank [4], respectively, before DAD has been promoted to an important concept in the treatment of irradiation damage in non-cubic metals by Woo $[5,6]$.

The role played by DAD in the irradiation-induced transformation of a void ensemble from a randomly distributed collection to a highly ordered void lattice was summarized by Woo and Frank [7]: “... the one-dimensional migration of the (interstitial) crowdions... is in marked contrast to the three-dimensional diffusion of the dumbbell interstitials and the vacancies. This diffusional anisotropy difference between the crowdions and vacancies provides the source of ordering via a Darwinian selection among randomly distributed voids." Hence, the strength of DAD is important to the behavior of a void ensemble under irradiation, and at the same time, the evolution of the void ensemble reflects the 
nature and properties of the point defects introduced by irradiation.

In recent Monte Carlo simulations (MCS) of the behavior of void ensembles under irradiation [8-10], it is assumed that all or nearly all self-interstitials migrate onedimensionally along close-packed crystallographic directions over distances which are much larger than the average separation of interstitial sinks. This category of selfinterstitial atoms will be referred to as 1-D SIAs. By contrast, self-interstitials that change their directions of motion much more frequently behave as 3-D diffusers. Examples of perfect 3-D SIAs are the dumb-bell configurations in cubic metals, which may change their direction of motion after every elemental diffusion step.

In fcc metals, except $\mathrm{Au}$, the dumb-bell configuration is a self-interstitial ground state, whereas the crowdion configuration is an excited metastable state [11]. It is important to realize that although the crowdion population increases at the expense of the dumb-bell population as temperature increases, there is simultaneously a decrease in the 1-D character of the diffusion of the SIAs because the frequency of thermal conversions between the two interstitial configurations also increases quickly as temperature increases. This leads to a much shortened mean free path of crowdion diffusion in a given direction.

Using many-body potentials, Foster et al. [12] found that the self-interstitial ground states of bcc metals $\mathrm{Ta}, \mathrm{Cr}$, and $\mathrm{W}$ also took the crowdion configuration. Recent densityfunctional calculations of Derlet et al. [13] confirmed these findings for most bcc metals. Similar to fcc metals, temperature increase leads to a decrease of the fraction of 1-D SIAs. Due to an increase in the frequency of thermal reorientations to other close-packed directions as temperature increases, the crowdion mean free path in a given direction is also much shortened.

The strength of the DAD bias increases with the proportion of 1-D SIAs in the SIA population. Most void-swelling models that have stood the test of decades of experiments are implicitly based on 3-D SIA kinetics without the DAD bias. Void-lattice formation, on the other hand, requires the operation of the DAD bias associated with the 1-D SIA kinetics, as has been demonstrated convincingly by Woo and Frank [7]. Since the action of a large DAD bias may produce a very different void-swelling behavior [7], the apparent conflict has to be resolved. The fact is, as we will show in subsequent discussions, that unless the 1-D character of the SIAs is substantially reduced, so that the " 1 -D" features of the void ensemble are sufficiently mellowed, its "3-D" character cannot be stable. In this regard, Semenov and Woo [14] found that a sufficiently strong driving force for void-lattice formation can be provided by just a small fraction of 1-D SIAs. The subject of this paper is to determine quantitatively the fraction of 1-D SIAs that is sufficiently small to provide the near-3-D kinetics required for void swelling but, on the other hand, sufficiently large to produce the DAD effect required for void-lattice formation. Without loss of generality, we restrict ourselves to cubic metals.

\section{Background issues}

\subsection{Void-growth saturation due to 1-D SIAs}

When all self-interstitials are 1-D SIAs, the interstitial flux to a void is proportional to the void surface, whereas the flux of the 3-dimensionally diffusing vacancies to a void is proportional to the void radius [4]. Hence, the influx of SIAs into the voids increases faster than the vacancy influx when a critical void size is exceeded. With increasing void size the net vacancy influx decreases monotonically to zero, with the void radius finally saturating at a stationary value determined by the absorption cross section of dislocations for 1-D SIAs [4, 14, 15]. Further void growth gives rise to an increase of the interstitial absorption rate at the voids at the expense of the interstitial absorption at dislocations. In this way, the net influx of vacancies into voids is reduced to zero, and void growth comes to a halt.

When the 1-D SIA component is negligibly small, the evolution of a void ensemble is exclusively governed by the conventional dislocation bias [16]. In this case, the void ensemble remains random, and void growth does not saturate. The interesting case occurs when the 1-D SIA component is small but non-negligible, and the void sink strength $k_{c}^{2}$ is much larger than the dislocation density $\rho$, e.g., $\rho / k_{c}^{2} \sim 10^{-2}$. As pointed out in [14], void growth tends to saturate under such circumstances, and a random void distribution may become unstable. Indeed, in this case, a small 1-D SIA fraction of only $10^{-2}$ is still sufficiently large to induce a phase transition to form a void lattice. If selfinterstitials are predominantly of the 1-D SIA type, the void growth behavior will be dictated by DAD bias, and swelling will saturate irrespective of the spatial distribution of the void ensemble [4].

In the latter case, the ratio of the saturation radius of voids in a lattice to that in a random ensemble is related to the void lattice parameter by $\tanh ^{-1}(L / 2 \lambda)$ [14], where $L$ is the nearest-neighbor distance in the void lattice, and $\lambda$ is the mean free path of the 1-D SIAs between consecutive changes of their directions of motion. It is experimentally established that the sizes of voids in a lattice and in a random assembly at a given temperature are comparable, i.e., $\lambda \sim L$ [17-20]. This is in line with MCS results of Heinisch and Singh [8], who found that, for void-growth characteristics coming close to those observed experimentally, $\lambda$ should be less than $2 L$.

In contrast, the more recent MCS calculations of Heinisch and Singh [9] found that to produce a void lattice, the mean 
free path of the 1-D SIAs has to be at least about four times nearest-neighbor distance of the void-lattice, and the simulated void lattice formed is still far less well defined than the experimental ones even in this case. This result corresponds to a value of $\tanh (L / 2 \lambda) \cong 1 / 8$, which implies that random voids have to be much smaller in this case. This is a condition caused by the dominance of the 1-D migrating self-interstitials assumed, because with a smaller concentration of "1-D" SIAs, their mean free path $\lambda$ can be much larger than the average distance between voids without seriously affecting the behavior of the random void ensemble, as suggested in [14].

\subsection{Displacive instability due to 1-D SIAs}

The displacive stability of a void lattice in the case of 1-D SIA diffusion is another important issue, which has been investigated and confirmed by Woo and Frank [21]. However, Evans [10] found that a void asymmetrically located between nearest-neighbor voids in a close-packed direction receives unequal 1-D SIA fluxes through its opposite faces and thus is driven towards the closer of the two nearest neighbors. Accordingly, any incomplete void-lattice patches formed by chance would be destabilized as a result of coalescence, before a highly symmetrical, stable void lattice can develop.

This argument may seem plausible but has two major flaws. Firstly, at high-symmetry sites, where the coalescent forces are balanced [22], the probability of void nucleation is exponentially higher and may exceed by orders of magnitude the void nucleation probability in any other regions [14], including Evans' "relatively empty space" [23]. Secondly, by assuming that all or nearly all self-interstitials migrate one-dimensionally along close-packed crystallographic directions, Evans may have considerably underestimated the effect of void shrinkage arising from the stochastic nature of the point-defect fluxes to the voids. As a result, the lifetimes of all voids in Evan's calculation, irrespective of where they have been nucleated, could be much over-estimated, so that most voids last too long for coalescence to be important. It is indeed the proportion of 1-D SIAs, and hence the strength of DAD, which controls the relative strength of the two effects and thus the evolution of a void ensemble. Semenov and Woo showed that the probability of survival of a void against stochastic shrinkage depends exponentially on the net vacancy influx $[14,25]$. Obviously, this is in turn controlled by the proportion of 1-D to 3-D SIAs. Hence, the DAD strength drastically affects both the lifetime and the number density of the voids. For instance, a decrease of the 1-D SIA portion leads to a reduction of the void lifetime due to stochastic shrinkage, so that a void may disappear before it has a chance to coalesce with an aligned nearest neighbor void. In such a case, coalescence cannot significantly influence void-lattice formation.
In the subsequent detailed analysis of this paper it will be shown that, contrary to intuition, void nucleation is the least probable in this "relatively empty space" that is "unshaded" against point-defect fluxes and that stochastic void shrinkage caused by fluctuations of the point-defect fluxes due to the randomness of diffusion jumps and cascade initiation may compete very effectively against void coalescence [24-26]. Related issues will also be explored for various phases of evolution of the void-ensemble. In this investigation, vacancy emission from voids will be neglected, since in most metals, e.g., molybdenum [27], this process is not important at the temperatures at which void-lattice formation takes place [26].

\section{Void number density and size distribution of a random ensemble}

To clarify the foregoing issues, we first consider the effect of the strength of the DAD bias on the saturation void size and void-number density of a random void ensemble. The number density and size distribution of a void ensemble under irradiation is directly related to void nucleation, which essentially constitutes void growth from small void embryos. Since the evolution of small embryos can be significantly affected by the stochastic fluctuations, their effects cannot be neglected. Semenov and Woo [28, 29] derived a FokkerPlanck-type equation that describes the time evolution of a void ensemble via its size-distribution function $f(n, t)$ with the account of the stochastic effects due to the random nature of both the migratory jumps of point defects and cascade initiation:

$$
\begin{aligned}
\frac{\partial f(n, t)}{\partial t}= & -\frac{\partial}{\partial n}\left\{V(n)-\frac{\partial}{\partial n} D(n)\right\} f(n, t) \\
& +j_{0} \delta\left(n-n_{0}\right),
\end{aligned}
$$

where the size of a void is defined by the number $n$ of vacancies in the void. $V(n)$ is the mean-field-theory-type void growth rate, and $D(n)$ is the diffusivity that governs the "diffusive spread" of the void-size distribution due to stochastic fluctuations. $D(n)$ is related to the average point-defect fluxes and cascade properties and, in the absence of vacancy emission, has the following analytic form [28, 29]:

$$
\begin{aligned}
D(n) & =\frac{3 n^{1 / 3}}{2 a^{2}}\left(D_{v} C_{v}+D_{i} C_{i}\right)+\frac{3 G N_{d}}{4 a k}\left[1+\left(1-\varepsilon_{i}\right)^{2}\right] n^{2 / 3} \\
& \equiv d^{s} n^{1 / 3}+d^{c} n^{2 / 3} .
\end{aligned}
$$

Here $D_{j}$ and $C_{j}$ are respectively the diffusion coefficient and the concentration of the $j$-type point defects, $(j=i$ for the 3-D SIAs, $j=v$ for vacancies), $G$ is the effective generation rate of point defects, $N_{d}$ is the average number 
of point defects generated in a single cascade, $k^{2}$ is the total sink strength for three-dimensionally diffusing point defects, $a=(3 \Omega / 4 \pi)^{1 / 3}$, and $\Omega$ is the atomic volume, which in the following is taken to be $1.34 \times 10^{-29} \mathrm{~m}^{-3}$ corresponding to molybdenum. The superscripts $s$ and $c$ denote the two different origins of stochasticity considered here, namely, the random migratory jumps and random cascade initiations, respectively. Finally, $\left(1-\varepsilon_{i}\right)$ is the fraction of mobile 3-D SIAs. For convenience, we assume in the present paper that all other self-interstitials are 1-D SIAs, i.e., there are no immobile SIA clusters, or, in the terminology of Woo and Singh [30], the production bias is switched off. Under these circumstances, $\varepsilon_{i}$ is a measure of the strength of DAD.

Since the total void-number density must be finite and small vacancy clusters consisting of two or three vacancies are mobile, i.e., voids below a minimum size $n_{\min }$ are considered to have exited the void ensemble, the kinetic equation (1) has to satisfy the following boundary conditions:

$f\left(n=n_{\min }, t\right)=0, \quad f(n \rightarrow \infty, t) \rightarrow 0$.

Three-dimensional vacancy clusters which are still small $\left(n \sim n_{\min }+1\right)$ but immobile (microvoids) are homogeneously produced either directly in the collision cascades [31-33] or via the consecutive agglomeration of single vacancies. This is taken into account by the source term $j_{0}$ in (1), where the $\delta$-function represents the assumption that initially all microvoids are of the same size $n_{0}\left(=n_{\min }+1\right)$.

The conditions when DAD bias is a major driving force for void growth are investigated in [14]. Following that reference, the mean-field void growth rate $V(n)$ governed by the 1-D SIAs can be written in terms of the mean-field void saturation size $n_{s}$ as

$V(n)=\frac{3 n^{1 / 3} G \varepsilon_{i}}{a^{2} k^{2}}\left(1-\frac{n^{1 / 3}}{n_{s}^{1 / 3}}\right) \equiv v n^{1 / 3}\left(1-\frac{n^{1 / 3}}{n_{s}^{1 / 3}}\right)$.

A void larger than $n_{s}$ absorbs more interstitials than vacancies. The stationary void-size distribution $f(n)$ from the solution of (1) satisfying $n \geq n_{0}$ and the boundary conditions (3) is given by [14]

$$
\begin{aligned}
f(n) & =\frac{j_{0}\left(n_{0}-n_{\min }\right)}{D(n)} \exp \left[\int_{n^{*}}^{n} \frac{V\left(n^{\prime}\right)}{D\left(n^{\prime}\right)} d n^{\prime}\right] \\
& =\frac{j_{0}\left(n_{0}-n_{\min }\right)}{\nu n_{s}^{1 / 3}} \frac{\chi\left(n / n_{s}\right)}{n_{s}},
\end{aligned}
$$

where

$$
\begin{aligned}
\chi(x) & =\frac{\exp \left\{\left[I_{1}(x)-I_{2}(x)-I_{1}\left(n^{*} / n_{s}\right)+I_{2}\left(n^{*} / n_{s}\right)\right] / \gamma_{c}\right\}}{\left(\gamma_{s} x^{1 / 3}+\gamma_{c} x^{2 / 3}\right)} \\
n_{\min } & \leq n^{*} \leq n_{0},
\end{aligned}
$$

$$
\begin{aligned}
I_{1}(x)= & \frac{3}{2}\left(\frac{\gamma_{s}}{\gamma_{c}}\right)^{2}\left[\left(\frac{\gamma_{c}}{\gamma_{s}} x^{1 / 3}-1\right)^{2}+2 \ln \left(\frac{\gamma_{c}}{\gamma_{s}} x^{1 / 3}+1\right)\right] \\
I_{2}(x)= & \left(\frac{\gamma_{s}}{\gamma_{c}}\right)^{3}\left(\frac{\gamma_{c}}{\gamma_{s}} x^{1 / 3}-1\right)^{3}+\frac{3 \gamma_{s}}{2 \gamma_{c}} x^{2 / 3} \\
& -3\left(\frac{\gamma_{s}}{\gamma_{c}}\right)^{3} \ln \left(\frac{\gamma_{c}}{\gamma_{s}} x^{1 / 3}+1\right) .
\end{aligned}
$$

The parameters $\gamma_{s}$ and $\gamma_{c}$ are related to $d^{s}$ and $d^{c}$ in (2) according to

$\gamma_{s}=\frac{d^{s}}{v n_{s}} \cong \frac{1}{\varepsilon_{i} n_{s}}$

$\gamma_{c}=\frac{d^{c}}{v n_{s}^{2 / 3}}=\frac{a k N_{d}}{4 \varepsilon_{i} n_{s}^{2 / 3}}\left[1+\left(1-\varepsilon_{i}\right)^{2}\right]$.

From (5) one can readily calculate the void number density $N_{c}$, the void sink strength $k_{c}^{2}$, and the swelling $S$ of the stationary state as a function of $\varepsilon_{i}$ and $n_{s}$ :

$$
\begin{aligned}
\frac{4 \pi N_{c} r_{s}}{\Omega k^{2}} & =\frac{j_{0}}{\varepsilon_{i}} \int_{n_{0} / n_{s}}^{\infty} \chi(x) d x, \\
\frac{k_{c}^{2}}{k^{2}} & =\frac{j_{0}}{\varepsilon_{i}} \int_{n_{0} / n_{s}}^{\infty} x^{1 / 3} \chi(x) d x \leq 1, \\
\frac{3 S}{k^{2} r_{s}^{2}} & =\frac{j_{0}}{\varepsilon_{i}} \int_{n_{0} / n_{s}}^{\infty} x \chi(x) d x .
\end{aligned}
$$

In (11) to (13), $r_{s}=a n_{s}^{1 / 3}$, the difference $n_{0}-n_{\min }$ is taken to be unity, and, if one may neglect the formation of microvoids by the agglomeration of single vacancies, the spatially homogeneous rate of microvoid generation $j_{0}$ is simply given by $q G / N_{d}$, where $q$ is the formation probability of small immobile three-dimensional vacancy clusters in collision cascades. Thus, through the dependence of $V(n)$ on $\varepsilon_{i}$ in (4), one can already see that the number density and sizedistribution of the void ensemble depend on the strength of DAD.

The right-hand side of (12) can be expressed as a function of the total void-number density $N_{c}$, using the ratio of expressions (11) and (12). This function is shown in Fig. 1a for various values of $n_{s}$ (or the corresponding radius $r_{s}$ ) and the fractions $\varepsilon_{i}$ of 1-D SIAs. According to experimental observations in molybdenum [17, 34], niobium [19], and nickel [35], the voids dominate the point-defect sinks during the process of void-lattice formation. Therefore, in Fig. 1a the actual void concentrations for various parameter sets can be read off from the intersections of the corresponding curves with the horizontal line $k_{c}^{2} / k^{2}=1$.

When the proportion of 1-D SIAs varies between its extremes, qualitative change of the saturation void size occurs. As already mentioned in Sect. 1.1, for a SIA population that is predominantly 1-D SIAs, the saturation void 

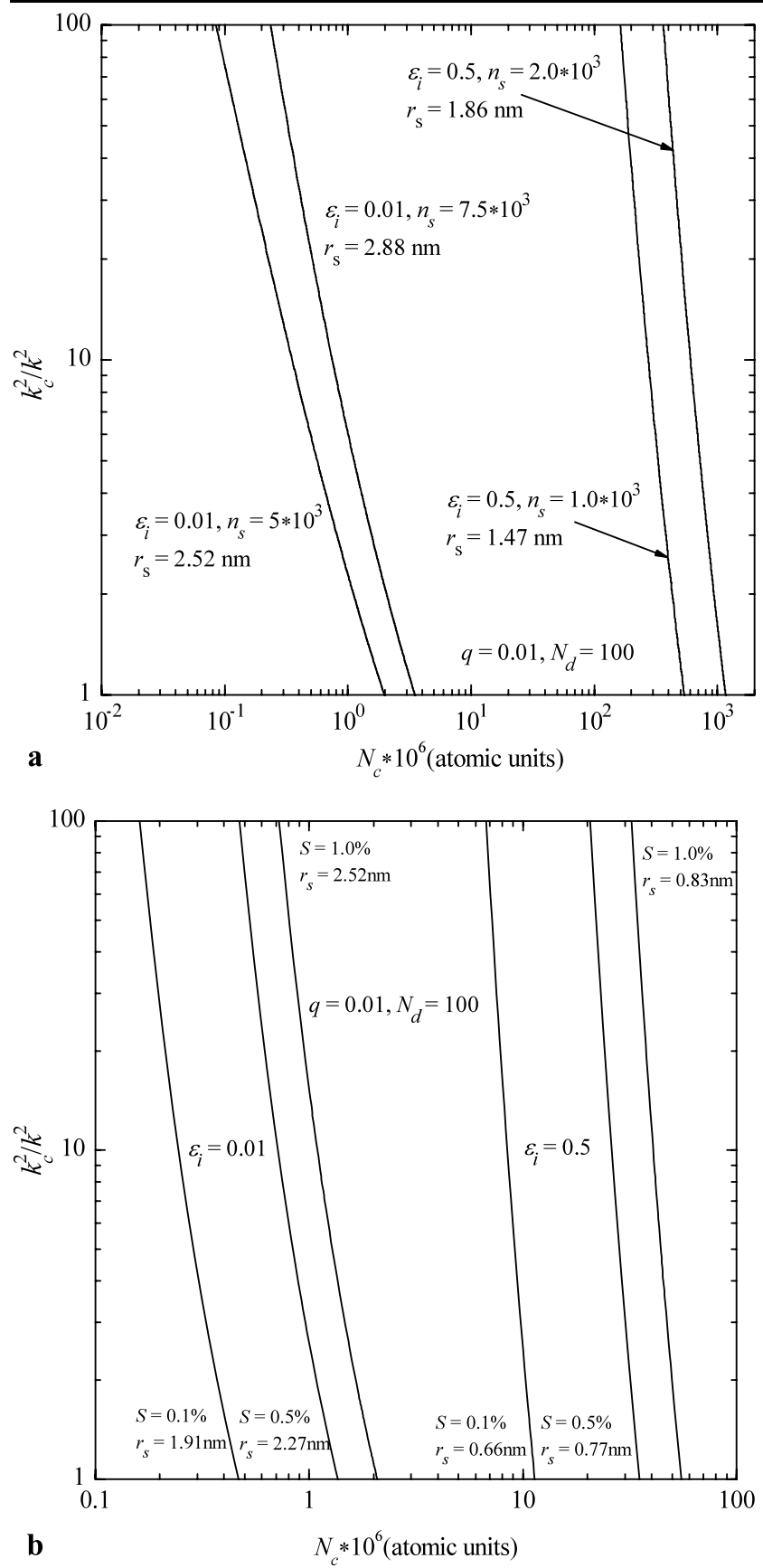

Fig. 1 (a) $k_{c}^{2} / k^{2}$ (from (12)) as a function of the total void number density $N_{c}$ for various values of $\varepsilon_{i}$ and $r_{s}$ (or $n_{s}$ ). (b) The same as (a) for various values of the total void swelling $S$

size is determined by the absorption cross section of dislocations for the 1-D SIAs [14, 15]. On the other hand, it was also shown that if the fraction of 1-D SIAs is small, the mean-field void growth would not saturate until the sink strength of voids becomes much larger than that of dislocations [14]. Before that stage is reached, the DAD effect is negligible, and the void growth is governed by the conventional dislocation bias, and the 1-D SIAs do not

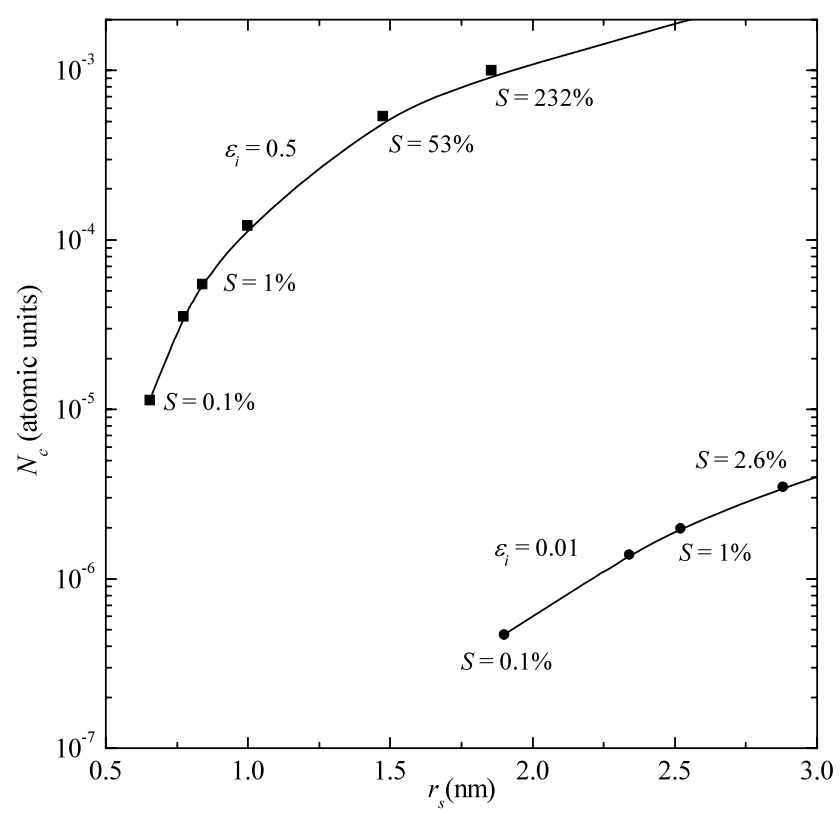

Fig. 2 Using the results of Figs. 1a and $1 \mathrm{~b}$, the total void number density $N_{c}$ is plotted as a function of $r_{s}$ for various values of $\varepsilon_{i}$. The corresponding swelling for some data points is also shown

have significant effects on the evolution of the void ensemble. According to [14], as the void sink strength increases and exceeds the dislocation sink strength, void growth progressively slows down and eventually stops. The saturation void size depends on the accumulated swelling $S$ as a result.

In Fig. $1 \mathrm{~b}, k_{c}^{2} / k^{2}$ is plotted as a function of the void number density for various values of $S$ according to (12). Similarly to Fig. 1a, the corresponding void concentration $N_{c}$ and saturation void size $n_{s}$ are determined from the intersections of the corresponding curves with the $k_{c}^{2} / k^{2}=1$ line. According to this figure, the increase in swelling is more due to the increase of $N_{c}$, i.e., nucleation of new voids, than the increase of $n_{s}$, i.e., growth of existing ones. This trend is independent of the values of $\varepsilon_{i}$ and only reflects that the growth of smaller voids is favored over the larger ones due to the quadratic increase of 1-D SIA absorption with void size.

We summarize the results in Figs. 1a and 1b in Fig. 2, where we plot the void-number density $N_{c}$ as a function of the mean-field saturation radius $r_{s}$, for the two values of $\varepsilon_{i}(0.5$ and 0.01$)$. The corresponding values of saturation swelling for the data points are also shown. It is clear from Fig. 2 that the characteristics of the void ensemble are very sensitive to the strength of DAD as measured by the value of $\varepsilon_{i}$. For a strong $\operatorname{DAD}\left(\varepsilon_{i}=0.5\right)$, a super-high void-number density of $\sim 10^{26} \mathrm{~m}^{-3}$ is required to satisfy saturation sizes even as small as $r_{s}=1.5$ to $2.0 \mathrm{~nm}$. Even with such small voids, the saturated swelling $\left(S \cong N_{c} n_{s}\right)$ would be on the order of $100 \%$, much too large in comparison with experi- 
mental void-swelling in this temperature range, which seldom exceeds a few percent $[17,19,20,27,34,36]$. For a weak DAD corresponding to $\varepsilon_{i} \sim 10^{-2}$, on the other hand, Fig. 2 shows that void number densities of $10^{22}-10^{23} \mathrm{~m}^{-3}$ $\left(\sim 10^{-6}\right.$ atomic unit) are easily obtained with saturation void radii of $\sim 2 \mathrm{~nm}$, producing swellings on the order of only a few tenths of $1 \%$. This is typical of experimental observations at temperatures for which vacancy emission is negligible [17, 20, 27, 37].

The ultra-high total void-number density for strong DAD is understandable when the probability of void survival is taken into account. This probability can be described in terms of the characteristic times $\tau_{D} \approx n^{2} / D(n)$ and $\tau_{V} \approx$ $n / V(n)$ via the ratio $\tau_{D} / \tau_{V}$, where $\tau_{D}$ and $\tau_{V}$ are respectively the characteristic times of stochastic void shrinkage and void growth due to net vacancy influx (see (5)). The value of $\tau_{D} / \tau_{V}$ is directly proportional to $\varepsilon_{i}$, a measure of the DAD strength. A high value of $\tau_{D} / \tau_{V}$ indicates a high probability of void survival. For a value of $\varepsilon_{i}=0.5$, the net mean-field vacancy flux to the smaller voids is so high that $\tau_{V}$ is much shorter than $\tau_{D}$, producing a driving force for the growth of microvoids, which far exceeds the stochastic forces that drive their shrinkage. It is the resulting high void nucleation rate that produces the high voidnumber density.

It should be noted that the value of $q=10^{-2}$ used in these calculations means that in 100 cascade events only one immobile three-dimensional vacancy cluster is created on the average. This assumption already corresponds to the low end of the range of results obtained in molecular-dynamics simulations of collision cascades [31, 38, 39]. It is clear from Fig. 1a that the unrealistically high values of swelling would still be unavoidable even if a $q$-value further reduced by an order of magnitude is used instead.

In Fig. 3, the void-size distributions for strong and weak DAD are also shown. For the case of strong DAD $\left(\varepsilon_{i}=0.5\right)$, the distributions have very narrow peaks near tiny saturation sizes. Note that these peaks are much sharper than what appears because the distribution functions for this case are scaled down by a factor of 50. Such distributions represent a uniform void size and thus are not consistent with the much broader void size distributions observed in experiments $[17,19,37]$. These very sharp peaks in the void size distributions as well as the high void number densities are due to the dominance of mean-field void growth over the broadening effect of the stochastic fluctuations. In terms of the characteristic times, the time $\tau_{V}$ a void needs to grow to its saturation size is significantly shorter than the time $\tau_{D}$ to shrink away by stochastic fluctuations.

Within the same framework, it is found that, for a weak DAD $\left(\varepsilon_{i} \sim 10^{-2}\right)$, the influence of the stochastic fluctuations is enhanced exponentially with increasing $\tau_{V}$. As a result, only a much smaller number of microvoids manage

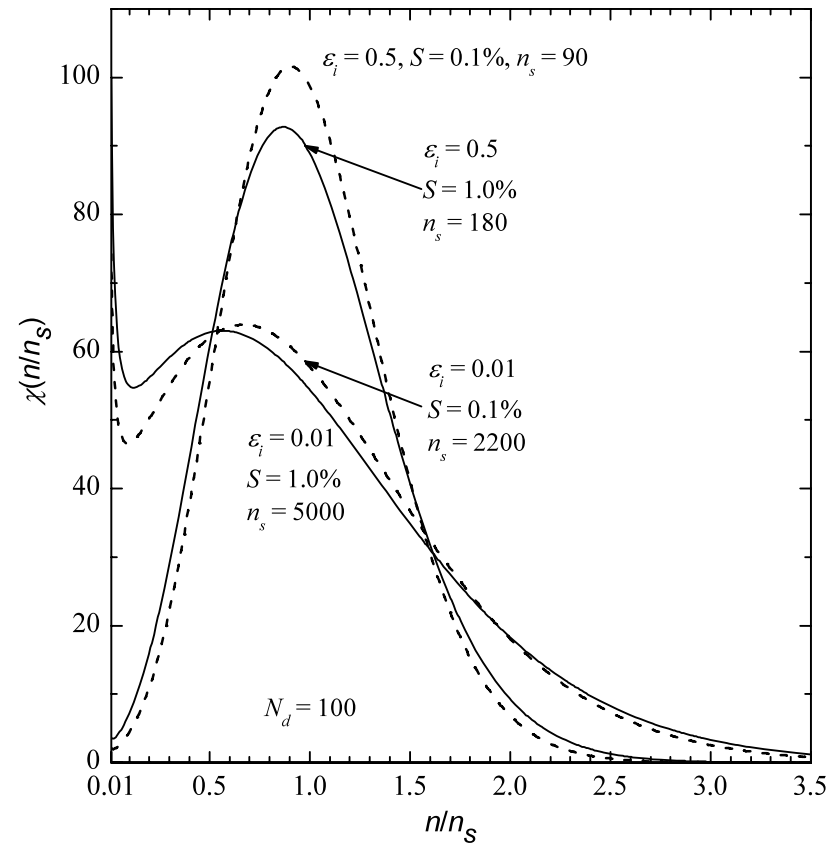

Fig. 3 Void size distribution functions for various values of $\varepsilon_{i}$ and $S$. For $\varepsilon_{i}=0.5$, the distribution functions are scaled down by a factor 50 , i.e., $\chi\left(n / n_{s}\right) / 50$ is presented

to survive (i.e., the total void number density is drastically reduced), and the void size distributions become distinctly broader, resembling those observed experimentally.

In view of the fact that most void-swelling models that have stood the test of decades of experiments are implicitly based on 3-D SIA kinetics without the DAD bias, the findings in this section is hardly surprising, namely, that only a sufficiently weak DAD can be consistent with the evolutionary characteristics of a random void ensemble. This is a very important point to realize in view of the recent renewed interest on the low-dimension migration of self-interstitials and their clusters.

Since both the growth and coalescence of voids are related to the influx of 1-D SIAs, when DAD is reduced, the stochastic fluctuations do not only affect the void size evolution but also their alignment and coalescence. The nucleation of voids in the early stages of void-lattice formation and their subsequent evolution in regard to coalescence will be considered in the following sections, where, unless stated otherwise, a weak DAD, viz., $\varepsilon_{i}=10^{-2}$, is assumed.

\section{Void alignment}

From (1) the evolution equation of the void-number density $N_{c}(t)$ can be written as:

$$
\frac{d N_{c}(t)}{d t}=j_{0}-\left[\frac{\partial}{\partial n} D(n) f(n, t)\right]_{n=n_{\min }} .
$$


Stationary condition $\left(d N_{c} / d t \rightarrow 0\right)$ is attained when the nucleation of new voids is balanced by the destruction of existing ones due to stochastic shrinkage. As it can be seen in Fig. 2, the number density of a random void ensemble increases with the saturation void size $n_{s}$. This reflects the general rule that the larger the voids are, the smaller is the effect of stochastic fluctuations on their evolution. Indeed, the ratio of characteristic times $\tau_{V} / \tau_{D}$, as expressed by the parameters $\gamma_{c}$ and $\gamma_{s}$, decreases with increasing saturation size $n_{s}$. According to (6), this means that the probability of void loss by stochastic shrinkage decreases exponentially as the size of void increases. Thus, even after the density of randomly distributed voids has reached saturation, successful nucleation of voids is still likely to take place in regions where conditions facilitate their growth to sizes larger than those of the randomly situated voids. The total volume of such regions may be relatively small, but since the density of voids in these regions increases exponentially with saturation size, it may be sufficiently large so that the swelling in these regions dominates that of the entire void ensemble. Indeed, if $q_{s}$ is the total volume fraction of regions where void growth saturates at a size $n_{s}$, then the generation rate of microvoids in these regions is equal to $j_{0} q_{s}$. Figure 4 shows the calculated $q_{s}$ as a function of the saturation void radius for different values of void swelling. One can see that $q_{s}$ may be very small, even well below $1 \%$. Since the calculations for Fig. 4 follow the procedure described in Sect. 2, which does not take into account that voids may actually have different saturation sizes, the results presented in Fig. 4 are, strictly speaking, applicable only when the void ensemble is dominated by the voids with certain saturation size and in the other cases should only be considered as an estimation. In the following, the issue of competition between the subsystems of voids characterized by different saturation sizes is studied in more detail.

\subsection{Initial void alignment}

The stationary state of a random void distribution, as defined by $d N_{c} / d t \rightarrow 0$ in (14), is dynamically stable only conditionally. In other words, a random void distribution may become unstable, and disorder/order transition of the system may take place due to the resulting bifurcation. Semenov and Woo derived the instability condition and showed that it can only be fulfilled when the DAD is neither overwhelmingly large nor negligibly small [14]. In the following, we consider the evolution of the void ensemble after its random distribution becomes unstable.

According to [14], the saturation radius $r_{s}(\mathbf{x})$ of a void at location $\mathbf{x}$ can be written as

$r_{s}(\mathbf{x}) \cong \frac{2 \pi \sum_{c} r_{c}^{2} \sum_{\alpha} \tanh \left(L_{\alpha}\left(\mathbf{x}_{c}\right) / 2 \lambda\right)}{M \phi(\mathbf{x}) k_{c}^{2}}$

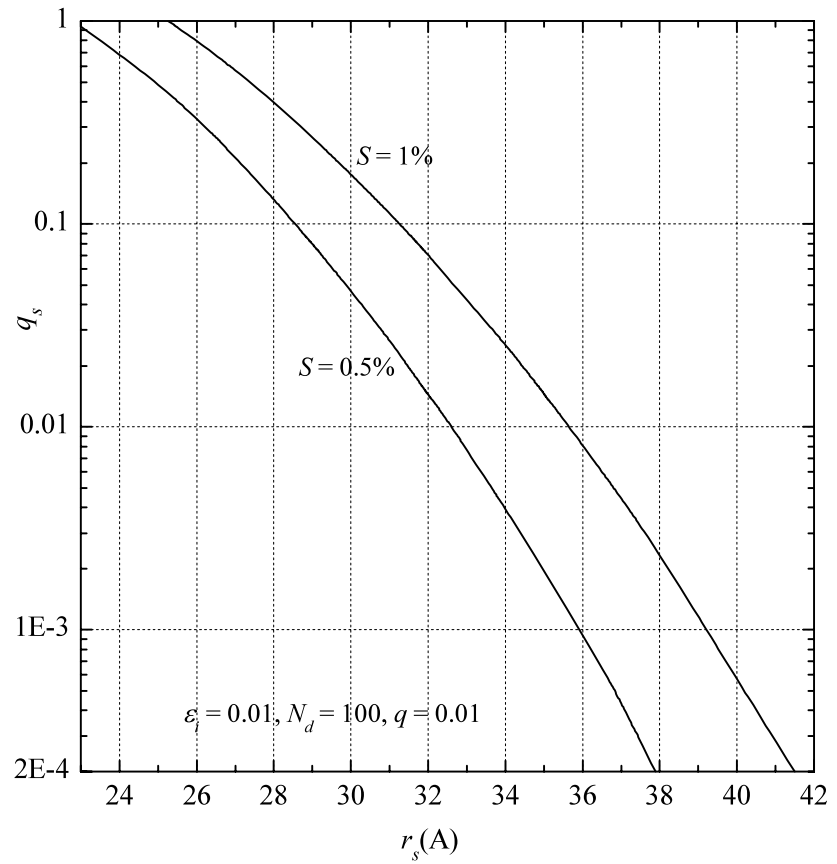

Fig. 4 Volume fraction $q_{s}$ as a function of the void saturation size $r_{s}$ for various values of total void swelling $S$

where

$\phi(\mathbf{x})=\frac{\sum_{\alpha} \tanh \left(L_{\alpha}(\mathbf{x}) / 2 \lambda\right)}{2 M}$,

$L_{\alpha}\left(\mathbf{x}_{c}\right)$ is the separation of the $c$ th void with radius $r_{c}$ from its nearest neighbor along the close-packed direction $\alpha(1 \leq$ $\alpha \leq 2 M), M$ is the number of close-packed directions of the crystal, and the sums run over all existing voids and both the left and right sides of all close-packed directions.

In an ensemble of random voids, the mean distance $L_{1}$ between two voids along a close-packed direction is equal to $2 \Omega / \pi r_{s}^{2} N_{c}$, which can be written in terms of the swelling $S \cong 4 \pi r_{s}^{3} N_{c} / 3 \Omega$ as $L_{1} / \lambda=\left(2 / \pi^{1 / 3}\right)(4 / 3 S)^{2 / 3} \times$ $\left(\Omega / N_{c}\right)^{1 / 3} / \lambda$. For void swelling of less than $1 \%[17,20$, $27,34,37]$, the values of $\tanh \left(L_{1} / 2 \lambda\right)$ and hence $\phi$ are close to unity, even if the mean free path $\lambda$ of 1-D SIAs is much larger than the average distance between voids $\left(\lambda\left(N_{c} / \Omega\right)^{1 / 3} \sim 10\right)$. Voids in the ensemble are likely to receive the full flux of suitably oriented 1-D SIAs produced within its interstitial supply cylinders (ISCs) [4]. They do not share these 1-D SIAs with the other nearest neighbor voids. Figure 5 illustrates the supply cylinder concept for non-overlapping cylinders. As a result, (15) can be expressed as $r_{s}=\left\langle r_{c}^{2}\right\rangle /\left\langle r_{c}\right\rangle$ for a truly random distribution of voids, where $\left\langle r_{c}\right\rangle$ is the average radius of these voids, and $\left\langle r_{c}^{2}\right\rangle$ is the average square radius.

Voids with overlapping ISCs (Fig. 6), on the other hand, can grow to sizes larger than the randomly situated ones because the interstitial influx per void is reduced by the 


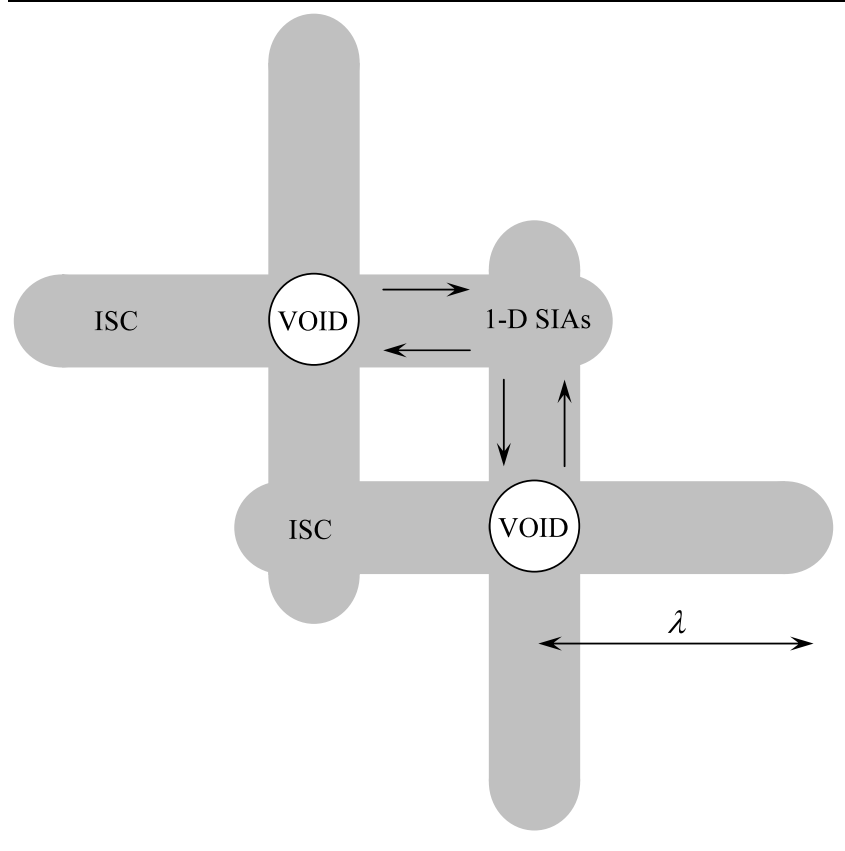

Fig. 5 Illustration of interstitial supply cylinders of a void. 1-D SIA created between two voids separated in its traveling direction by the distance $l$ will combine with one of the voids with a probability $(2 \lambda / l) \tanh (l / 2 \lambda)$. Thus, most of the 1-D SIAs annihilated at a void come from a cylindrical region of length $\lambda$ extending from the void surface along the close-packed directions. There are eight supply cylinders for each void in a bcc lattice and twelve in an fcc lattice

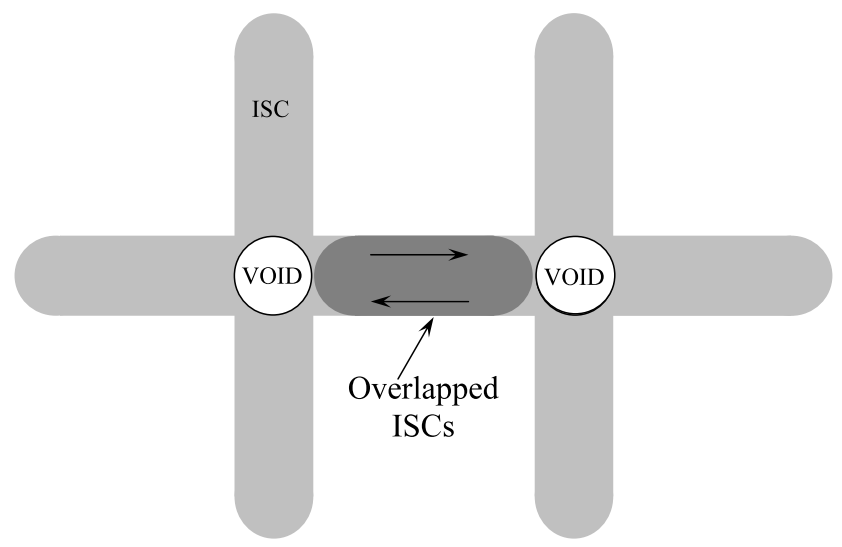

Fig. 6 When the separation of two voids along a close-packed direction is sufficiently small $(l \sim \lambda)$, their ISCs overlap or an ISC of the one void even protrudes into the other void. Thus, a reduction of the on supplies to both voids occurs $(\tanh (l / 2 \lambda)<1)$. As a result, the net vacancy fluxes to both voids increase, which leads to an enhanced growth of these voids

sharing between neighboring voids of the 1-D SIAs generated in the overlapping ISCs. Indeed, compared to cases where there is no effective ISC overlap, $\tanh \left(L_{\alpha} / 2 \lambda\right)<1$ in at least one of the close-packed directions, and thus $\phi<1$. Therefore, after the density of randomly distributed voids has reached saturation $\left(d N_{c} / d t \rightarrow 0\right)$, void nucleation (along the close-packed directions) is still possi- ble. Nevertheless, this does not mean that these voids will automatically arrange themselves strictly in rows, as erroneously assumed by Evans [23]. After the nucleation of an aligned void in a given close-packed direction, the next void may form in a different, yet equivalent, close-packed direction. Moreover, while for the random distribution of small voids, the average distance $L_{1}$ between two voids along the close-packed directions is much larger than the average distance $L_{3}=\left(3 \Omega / 4 \pi N_{c}\right)^{1 / 3}$ in three dimensions, for a closepacked plane defined by two nonparallel close-packed directions, the corresponding distance $L_{2}$ on the plane is

$L_{2}=\left(\frac{\Omega}{2 \pi N_{c} r_{s}}\right)^{1 / 2} \cong\left(\frac{\Omega}{\pi N_{c}}\right)^{1 / 3}\left(\frac{1}{6 S}\right)^{1 / 6}$.

Hence, the ratio $L_{2} / L_{3}$ decreases with increasing swelling $S$. When $S$ is larger than $0.5 \%, L_{2} / L_{3}$ is less than 2 . Thus, for randomly distributed voids, $L_{1}$ is in general much larger than $L_{2}$ and $L_{3}$.

Keeping the preceding discussion in mind, a "thoughtexperiment" illustrated in the series of void nucleation events in Fig. 7a-g can be used to speculate how the initiation of void alignment may occur. Let us consider two nonparallel close-packed directions (dashed lines) running through two neighboring voids (solid circles) on a closepacked plane. Referring to Fig. 7a, the intersections (open circles) of these directions mark potential nucleation sites for voids having two overlapping ISCs. Note that the average distance between a potential void and a nearest neighbor along any out-of-plane close-packed direction is equal to $L_{1} / 2$, which is much larger than $L_{2}$, so that ISC overlap in those directions is unlikely.

For random void distributions, the only locations which may serve as sites for further nucleation are locations at which voids can have one or two overlapping ISCs. The latter ones are more favorable because there voids are able to grow to larger sizes and are thus more resistant to stochastic shrinkage (see also Sect. 3.2). As a consequence, early void ordering in the initial stages of void lattice formation is more likely to occur on closed-packed planes rather than along close-packed directions, which is in agreement with observations [23]. Obviously, the intuitive picture that onedimensional SIA transport must result in linear initial alignment of randomly arranged voids is uncorroborated.

Figure 7 shows two out of many potential ways how, starting from a random void distribution, planar void ordering may evolve. Note that preexistence of final void lattice sites is not assumed. According to this series of figures, locations where voids may nucleate with more than two overlapping ISCs already emerge after the first round of alignedvoid nucleation on the close-packed planes (Fig. 7a). While planar ordering proceeds, potential nucleation sites for voids with an increasing number of overlapping ISCs consecutively come into existence, and, as a result of planar ordering 
Fig. 7 Illustration of planar void ordering due to the nucleation of voids in a close-packed plane at the intersections of corresponding close-packed directions (dashed lines). From (a) to (d), new voids (open symbols) with a greater number of overlapping ISCs nucleate, while already existing voids (closed symbols) with a smaller number of overlapping ISCs shrink away. Figures (e) to (g) demonstrate that locations where voids survive are not fixed; rather they depend on where nucleation is possible, i.e., on the locations of aligned nearest neighbor voids. As required by the condition of swelling saturation, the number of voids remains the same through all the figures

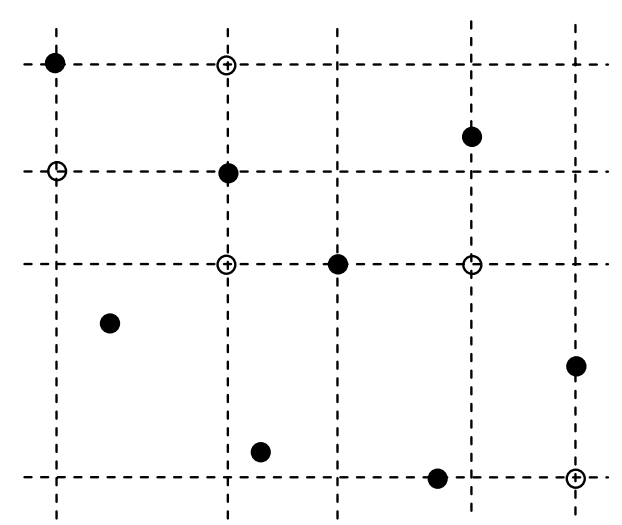

$\mathbf{a}$

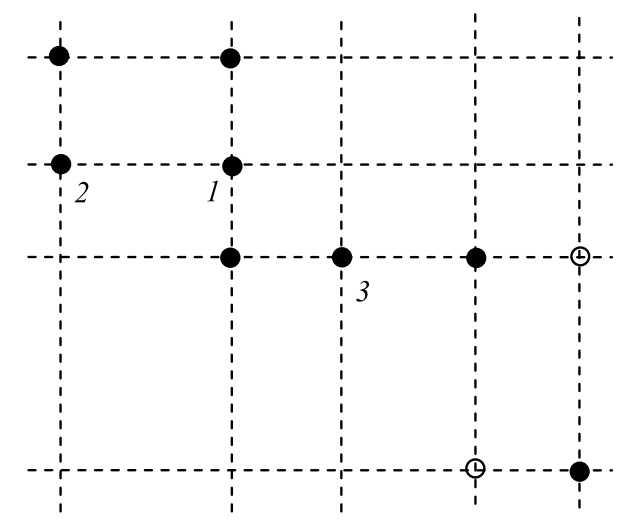

b

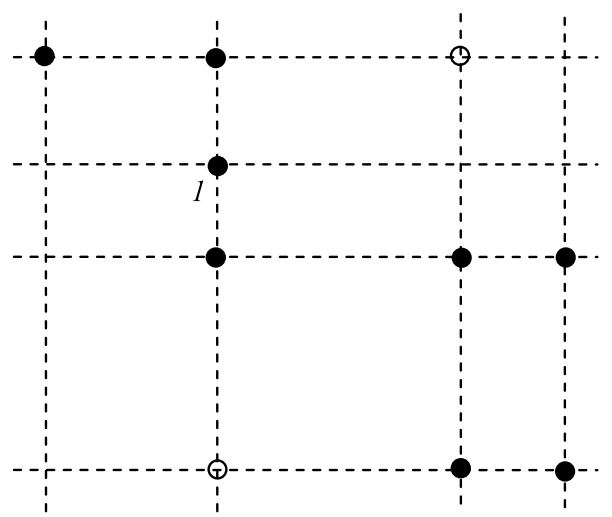

c

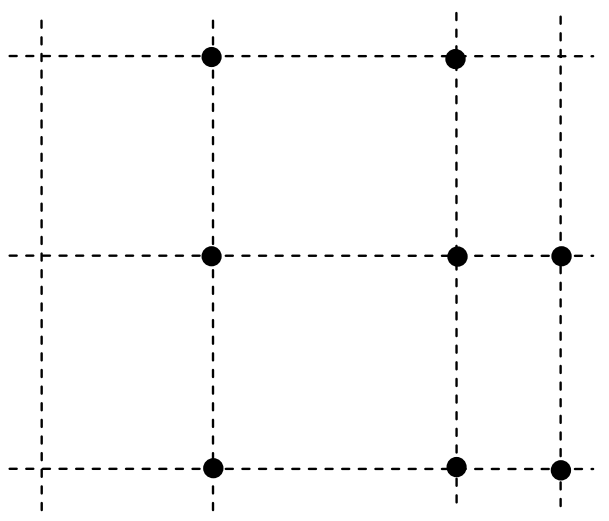

d

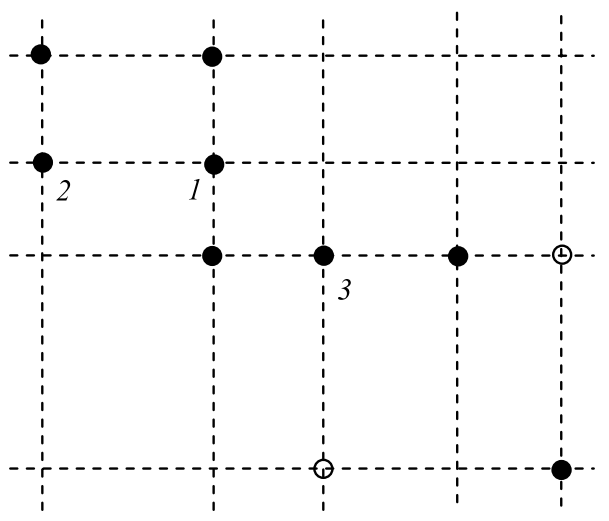

e

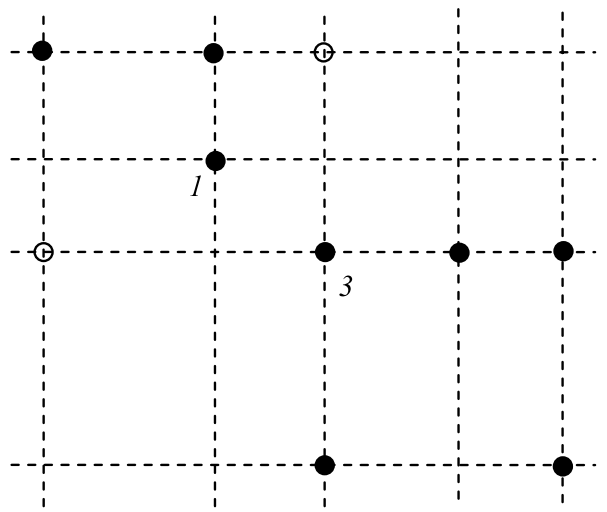

f

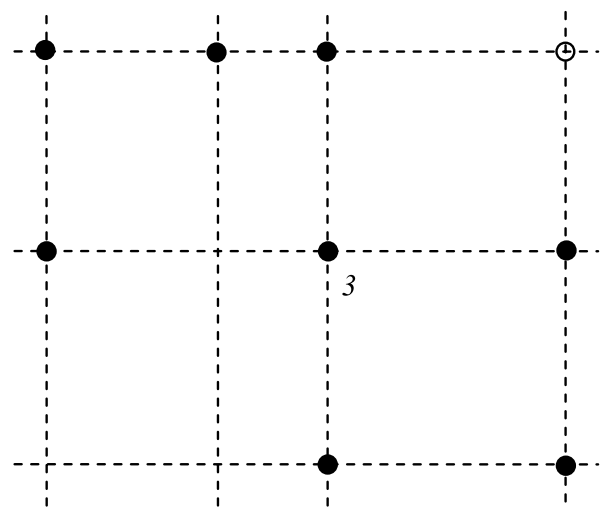

g 
on all close-packed planes, regular void-lattice sites eventually appear. Finally, subsequent nucleation of voids on these sites leads to the completion of the void lattice.

\subsection{Shrinkage of voids in non-lattice positions}

For a well-defined void lattice to form, nucleation and growth of new voids at the void-lattice positions is only necessary but not sufficient. The annihilation of voids, randomly positioned or left partially aligned during the intermediate stages of ordering, is equally important (Fig. 7). To describe this process we consider the Fokker-Planck equation

$\frac{\partial p(n, t)}{\partial t}=-\frac{\partial}{\partial n}\left\{V(n)-\frac{\partial}{\partial n} D(n)\right\} p(n, t)$

which governs the size evolution of an individual void $[28,29]$. The void-size probability distribution $p(n, t)$ satisfies the initial condition $p(n, t=0)=\delta\left(n-n_{0}\right)$ and the boundary conditions (3). Equation (18) follows from the kinetic equation (1) by dropping the source term $j_{0}$.

It may be readily shown that the stationary solution (5) can also be written as

$f(n)=j_{0} \tau(n)$,

where

$\tau(n)=\int_{0}^{\infty} p(n, t) d t$.

Thus, the stationary distribution function (5) is linearly related to the lifetime of a void with a size between $n$ and $(n+d n)$, the proportionality constant being $j_{0}$, the production rate of microvoids. The integration of $\tau(n)$ over all possible void sizes $n$ yields the average lifetime $\tau_{s}$. Consequently, the $r_{s}$ can be considered as a function of $\tau_{s}$, according to (5) to (10) and (19).

Physically, stochastic void shrinkage can be understood from the example of diffusion processes. In one dimension, if a random walker is not allowed to go to infinity, it will always revisit the starting point after some time. In the present case where void growth saturates, the size of every void in the ensemble fluctuates about a statistical mean but sooner or later returns to where it has started, i.e., to the size of a mobile vacancy/vacancy cluster, and ceases to exist as a void, or annihilates from the void ensemble. The average time spent by the void between nucleation and annihilation is the mean lifetime of the voids. Indeed, from (18) it can be easily shown that the probability for a void to eventually shrink away is exactly equal to one. Absent stochastic fluctuations, the shrinkage probability vanishes, and the void life-time becomes infinite.

In Fig. 8, $r_{s}\left(\tau_{s}\right)$ is presented as a function of $G \tau_{s}$ for different values of total sink strength $k^{2}$ or swelling $S$. In

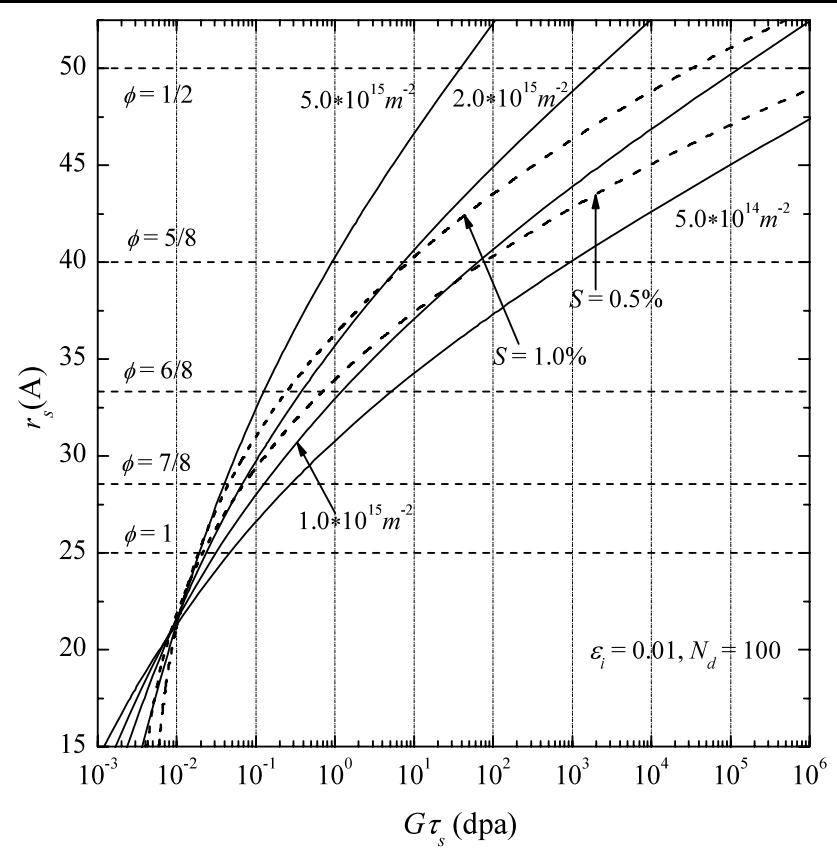

Fig. 8 Saturation void radius $r_{s}$ as a function of the mean lifetime dosage $G \tau_{s}$ of a void for various values of the total sink strength $k^{2}$ and the total void swelling $S$

the former case, the total sink strength can be due to voids of different saturation sizes, while in the latter case, all voids of the ensemble are assumed to have the same saturation radius $r_{s}\left(\tau_{s}\right)$. If we assume that the mean free path $\lambda$ is significantly larger than the average distance between voids as in the MC simulations, then, for the overlapping ISCs, $\tanh \left(L_{\alpha} / 2 \lambda\right) \ll 1$ and $\phi \cong(2 M-m) / 2 M$, where $m$ is the number of overlapping cylinders. Then in Fig. 8 the dashed horizontal lines labeled with different values of $\phi$ correspond to the saturation sizes of voids, which have $m=2 M(1-\phi)$ overlapping ISCs. For a bcc metal, $M=4$.

According to Fig. 8, the lifetime $K \tau_{s}$ of a random void $(\phi=1)$ and voids with one overlapping ISC $(\phi=7 / 8)$ are significantly shorter than the lifetime of voids with two overlapping ISCs $(\phi=6 / 8)$, which still does not exceed just a few NRT dpa. Here $K$ is the nominal NRT rate of displacements per atom (dpa) $(G / K \cong 0.3[33,39])$. Note that, for an initially random void distribution, the positions on a close-packed plane, where nucleating voids can have two overlapping ISCs, occupy a volume fraction of $q_{s} \approx 2 S$. Taking into account that $0.1<G \tau_{s} \leq 1$ dpa (Fig. 8), for $S=1 \%$, the number density of voids initially aligned in close-packed planes is $2 \operatorname{Sj}_{0} \tau_{s} \approx 2 \times 10^{22}$ to $10^{23} \mathrm{~m}^{-3}$. Since $r_{s} \cong 3.33 \mathrm{~nm}$, this means that more than half of the total swelling is due to voids aligned on the close-packed planes.

From Fig. 8 the lifetime of a void increases exponentially with its saturation size, and voids with several overlapping ISCs may survive extraordinarily long. However, the actual lifetime of a void is extremely sensitive to the specific local 
void arrangement. In Fig. 7b, void 1 with three overlapping ISCs may seem to stay much longer than the neighboring void 2 with only two overlapping ISCs. In reality, the disappearance of void 2 will eliminate one of the ISCs of void 1 , so that its annihilation will follow quickly after void 2 disappears. Thus, to a large extent, the lifetime of a local void arrangement is determined by the lifetimes of those voids in the arrangement, which have the smallest number of overlapping ISCs.

In other words, a spatial arrangement of voids survives the longest time if every void in the arrangement has the maximum number of overlapping ISCs with its nearest aligned neighbors. Obviously, such an arrangement has a form of lattice. Therefore, lattice voids have predominantly the longest lifetime and are the surviving species in a Darwinian selection in which the rules of survival are laid down by the DAD effect, as described by Woo and Frank [4, 7]. According to Fig. 8, the lifetimes of the lattice voids can practically be infinitely long. Because of the exponential dependence of the lifetimes on the microstructure and material characteristics via the parameter $\gamma_{c}$, the irradiation dose required for void lattice formation may vary considerably, ranging from 2 [27] to 400 NRT dpa [35].

Due to the continuous generation of microvoids in collision cascades, the shrinkage of existing voids on non-lattice sites during the void-lattice formation process does not mean that voids do not nucleate at these positions at all. Indeed, a subsystem of non-lattice voids may coexist with the void lattice at all times. However, if the parameter $\gamma_{c}$ for voids outside the lattice positions is larger than the critical value $\gamma_{c}^{c r} \approx 0.2$, the size distribution function $f(n)$ of these voids has a maximum at $n_{0}$ [14]. The evolution of these voids is dominated by stochastic fluctuations, and, as a result, during their short life, the size of these voids remains close to their embryonic size, $n_{0} \approx 4$ to 5 , which is well below the saturation size. Accordingly, microvoids generated outside lattice positions remain predominantly submicroscopic and do not affect the observable characteristics of the void ensemble. As it was previously pointed out [14], since the meanfield saturation size is a stable stationary point $\left(V\left(n_{s}\right)=0\right)$ in the evolution of the void ensemble, when the mean-field void size is no longer the most probable, a non-equilibrium phase transition in the void ensemble occurs. This transition is induced entirely by the stochastic noise in the point-defect fluxes [40].

Voids occupying positions between two lattice voids are able to reach larger saturation sizes than randomly arranged voids, because they have two overlapping ISCs. According to (15), their saturation size $r_{s}$ is given by

$r_{s} \approx \frac{M-2}{2 M}\left\langle\tanh \left(L_{\alpha} / 2 \lambda\right)\right| r_{c l}$, where $r_{c l}$ is the average size of lattice voids, and $\left\langle\tanh \left(L_{\alpha} / 2 \lambda\right)\right\rangle$ is the average of $\tanh \left(L_{\alpha} / \lambda\right)$ over all closepacked directions and all voids in the lattice.

In so-called hyperlattices, which are observed at elevated temperatures where vacancy emission from voids plays an important role in the growth of voids, the void-lattice parameters are indicative of the mean free path $\lambda$. For example, in $\mathrm{Nb}$ at $1010^{\circ} \mathrm{C}$ the void-lattice parameter is $146 \mathrm{~nm}$ [18], and in $\mathrm{Mo}$ at $1120^{\circ} \mathrm{C}$ it is $\sim 100 \mathrm{~nm}$ [34]. At the lower temperatures, where vacancy emission is negligible, the void-lattice parameters are considerably smaller, e.g., 20 to $30 \mathrm{~nm}$ in $\mathrm{Mo}$ and $\mathrm{Nb}[17,18,20]$. It is safe to assume that at lower temperatures, $\lambda$ is at least not smaller. This implies that at these lower temperatures the ratio $2 \lambda / L \sim 10$, corresponding to a value of $\tanh (L / 2 \lambda) \sim 0.1$ and $r_{s} \approx$ $M r_{c l} / 10(M-2)$. Since the largest lattice voids found at

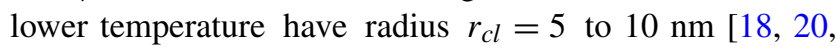
$35,36,41]$, the saturation size of non-lattice voids must be $r_{s}<1.5 \mathrm{~nm}\left(n_{s}^{1 / 3} \sim 10\right)$. With this value of $r_{s}$ and the expression $k_{c}^{2} \cong 3 S / r_{c l}^{2}$ for the sink strength of lattice voids, (10) yields

$\gamma_{c} \cong \frac{a \sqrt{3 S} N_{d}}{2 \varepsilon_{i} r_{c l} n_{s}^{2 / 3}} \approx \frac{2.5 \times 10^{-3}}{\varepsilon_{i}}>\gamma_{c}^{c r}$

for $S=1 \%$ and $N_{d}=100$. From this one may conclude that the upper limit of the fraction $\varepsilon_{i}$ of 1-D SIAs is about 1\%, which is in agreement with both findings of Sect. 2 and [14]. It is important to realize that the voids form a lattice only in this weak-DAD case. For a strong DAD $\left(\varepsilon_{i}=0.5\right)$, the random void distribution would be a stable stationary state. No disorder-order transition would take place. The void ensemble would evolve to a collection of small non-aligned voids $\left(r_{s}=1.47 \mathrm{~nm}\right)$ producing a swelling of $S \cong 50 \%$ with a sink strength of $7 \times 10^{17} \mathrm{~m}^{-2}$ (Figs. 1a and 2).

\section{Void coalescence}

Void coalescence is the result of the drift of unequally spaced voids along close-packed directions caused by the differential fluxes of 1-D SIAs received from opposite faces of the voids. Evans [10,23] has argued that void coalescence may constitute a serious deterrent to void lattice formation from an ensemble of random voids due to 1-D SIA diffusion. In the MC simulation [10], the velocity $v_{\alpha}$ of the "centre of gravity" of a void along a close-packed direction $\alpha$ can be calculated using the results of [14]:

$v_{\alpha}=\frac{3 \varepsilon_{i 0} G \lambda}{8 M}\left[\tanh \left(L_{\alpha l} / 2 \lambda\right)-\tanh \left(L_{\alpha r} / 2 \lambda\right)\right]$.

Here $\varepsilon_{i 0}$ is the fraction of crowdions produced directly in collision cascades, and $L_{\alpha l}$ and $L_{\alpha r}$ are the separations of 
the void along the close-packed direction $\alpha$ from its nearest neighbors on the left and right side, respectively. Note that in the case of crowdions the values of $\varepsilon_{i}$ and $\varepsilon_{i 0}$ are proportional but not necessarily equal to each other. It is found in [14] that for $\varepsilon_{i} \sim 10^{-2}, \varepsilon_{i 0}$ should not exceed about $10 \%$.

According to (23), the drift velocity of the void reaches its highest value for $L_{\alpha l} / 2 \lambda \gg 1$ and $L_{\alpha r} / 2 \lambda \ll 1$. This is the case if the left-side neighbor void is far away and thus the void (like void 2 in Fig. 7b) receives the full 1-D SIAs flux produced in its left ISC, whereas the 1-D SIAs flux produced in its right ISC have to be shared with the close-by neighboring void on its right side. The minimum time $t_{c l s}^{(1)}$ required for a void to drift over a distance equal to its radius $r_{s}$ is given by

$G t_{c l s}^{(1)}=\frac{8 M}{3 \varepsilon_{i 0}} \frac{r_{s}}{\lambda}$.

If a void is located between two other voids such that $L_{\alpha l} \sim$ $L_{\alpha r}$ and $L_{\alpha r} / 2 \lambda \ll 1$ (like void 3 in Fig. 7b), the corresponding time $t_{c l s}^{(2)}$ may readily be found from (23) as

$G t_{c l s}^{(2)}=\frac{8 M}{3 \varepsilon_{i 0}} \ln \left(1+\frac{r_{s}}{\Delta L_{0}}\right)$,

where $\Delta L_{0}$ is the initial deviation of the void from the center of the line connecting its nearest neighbor voids.

For $\lambda / r_{s} \approx 100, M=4$ (bcc lattice), and $\varepsilon_{i 0} \approx 0.1$, the minimum dose required for an aligned void to move over a distance equal to its own radius is $G t_{c l s}^{(1)} \approx 1 \mathrm{dpa}$, an order of magnitude larger than its total lifetime $G \tau_{s}$, which is smaller or about $0.1 \mathrm{dpa}$ (see $\phi=7 / 8$ in Fig. 8). Voids with two overlapping ISCs $(\phi=6 / 8)$ survive longer time $(0.1<$ $G \tau_{s} \leq 1 \mathrm{dpa}$ ). However, by (25) the irradiation dose needed for such voids to cover the one radius distance $\left(\Delta L_{0} \sim r_{s}\right)$ is almost two orders of magnitude larger $\left(G t_{c l s}^{(2)} \approx 74 \mathrm{dpa}\right)$ than their total life-time $G \tau_{s}$ from the creation in a cascade to the complete disappearance by shrinkage. Note also that, to coalesce with one of its neighboring voids, void 2 in Fig. $7 \mathrm{~b}$ needs to move out of the shade of another neighbor and lose one of its two overlapping ISC. Thus, voids that do not occupy lattice positions disappear long before they may coalesce with their aligned nearest neighbors. Lattice voids, on the other hand, have been shown to be stable with respect to coalescence despite their long-lifetime [21, 22].

As discussed earlier in this paper, the void lifetime increases exponentially with the value of $\varepsilon_{i}$. For $\varepsilon_{i} \sim 1$, as assumed in the MCS by Evans [10], it is much longer than the characteristic time for void coalescence. As a result, the loss of voids in those simulations is dominated by coalescence, and void lattice formation becomes difficult. Thus, in addition to not having taken into account void nucleation properly, Evans' conclusion that void-lattice formation cannot be explained in terms of 1-D SIA diffusion also suffers from having used a too large $\operatorname{DAD}\left(\varepsilon_{i} \sim 1\right)$ in his simulation, an assumption that does not corroborate other observations on void ensembles and swelling, as discussed in [14] and throughout the present paper.

\section{Conclusions}

The role of DAD in the evolution of a void ensemble under cascade irradiation is complex. The behavior of the void ensemble is very sensitive to the relative strengths of the pointdefect fluxes due to DAD compared to the stochastic fluctuations. Contrary to void growth driven by dislocation-bias, that driven by the DAD bias in the case of 1-D SIA diffusion saturates at a final void size. This is because the influx of 1-D SIAs into the voids is proportional to the void surface, whereas the vacancy influx is proportional to the void radius. Allowing for stochastic fluctuations of the point-defect fluxes arriving at a void, the finite void saturation radius implies that the void will be eventually annihilated by the stochastic shrinkage. The dynamic balance between the creation of new voids and the stochastic shrinkage of existing ones is the mechanism by which the steady-state voidnumber density is achieved.

The mean-field net vacancy flux received by a void that has not yet reached its saturation size is directly proportional to the DAD as measured by the fraction $\varepsilon_{i}$ of 1-D SIAs. An overestimation of $\varepsilon_{i}$ is magnified and thus gives rise to an exponential increase of the void lifetime because, with the increase of the mean-field growth rate, the relative importance of stochastic shrinkage is drastically quenched. This produces void-number densities and void swellings which are orders of magnitude larger than those observed. Conversely, if void swelling is forced to match the experimental values, the assumption of a high proportion of 1-D SIAs can only be satisfied for a random ensemble consisting of tiny voids of uniform below- $1 \mathrm{~nm}$ size at an extremely high number density, which is markedly different from what is observed. On the other hand, if a smaller proportion of 1-D SIAs is chosen $\left(\varepsilon_{i} \sim 10^{-2}\right)$, i.e., the DAD is reduced, the features of the corresponding void ensemble is in accordance with the experimental findings (Sect. 3). Thus, only a sufficiently weak DAD can be consistent with the evolutionary characteristics of a random void ensemble.

In the case of 1-D SIA diffusion, the spatial distribution of the void nucleation probability in an ensemble of randomly distributed voids is highly inhomogeneous. Contrary to intuition, locations surrounded by a few voids only are "unshaded" against the influx of 1-D SIAs, so that in these regions the void nucleation probability is low. Rather, voids tend to be nucleated at locations where the void density is higher, and thus the voids' supply cylinders for 1-D SIAs overlap, since in such regions the void lifetimes are higher 
by orders of magnitude. Although at low void swelling ( $S \leq 1 \%$ ) the volume fraction of regions containing overlapping ISCs is small, the number density of voids nucleated there can be sufficiently high to make these regions dominate the void ensemble. Because of this very pronounced inhomogeneity of the void nucleation probability, models not involving 1-D kinetics with these fundamental characteristics cannot be expected to successfully describe the spatial dependence of the microstructure evolution.

The stationary state of a random void distribution is dynamically stable if the DAD is either very strong or negligibly weak. Otherwise a random void distribution may become unstable under suitable conditions. When instability sets in, the firstly nucleating new voids, on the one hand, must be more stable against stochastic shrinkage than the already existing random voids and thus must have more overlapping ISCs but, on the other hand, cannot be expected to have, in general, more than two overlapping ISCs. Since voids with two (nonparallel) overlapping ISCs nucleate on close-packed planes determined by two different close-packed directions, initial void ordering is more likely to proceed on planes than along straight lines. During this planar ordering along all close-packed planes, locations with an increasing number of overlapping ISCs, and eventually the regular sites of the forming void lattice, emerge progressively. Since voids on lattice sites can have the maximum number of overlapping ISCs and, consequently, the largest saturation size, their lifetime is longer than those of any other voids, namely practically infinitely long.

After the void lattice has been formed, despite the continuous production of microvoids in collision cascades, additional nucleation of voids on non-void-lattice sites is suppressed, since, in the case of a small proportion of 1-D SIAs (e.g., $\varepsilon_{i} \sim 10^{-2}$ ), the lifetime of "interstitial" voids is stochastic-fluctuation-controlled and thus short. These voids spend most of their short life without exceeding their original microvoid size, i.e., they never come close to the saturation size and thus remain "invisible." In addition, contrary to what is true for $\varepsilon_{i} \sim 1$, for a small proportion of 1-D SIAs $\left(\varepsilon_{i} \sim 10^{-2}\right)$, the lifetime of voids on non-void-lattice sites is short compared to the characteristic time of void coalescence. Hence, stochastic shrinkage prevents such voids from coalescence and thus from destabilization of a partially formed void lattice.

Acknowledgements The authors are grateful for funding support by the Hong Kong Research Grant Council (PolyU 530507, 532204).

Open Access This article is distributed under the terms of the Creative Commons Attribution Noncommercial License which permits any noncommercial use, distribution, and reproduction in any medium, provided the original author(s) and source are credited.

\section{References}

1. U.M. Gösele, Prog. React. Kinet. 13, 63 (1984)

2. C.H. Woo, U. Gosele, J. Nucl. Mater. 119, 219 (1983)

3. C.H. Woo, J. Nucl. Mater. 120, 55 (1984)

4. C.H. Woo, W. Frank, J. Nucl. Mater. 137, 7 (1985)

5. C.H. Woo, ASTM STP 955, 70 (1987)

6. C.H. Woo, J. Nucl. Mater. 159, 237 (1988)

7. C.H. Woo, W. Frank, Mater. Sci. Forum 15-18, 875 (1987)

8. H.L. Heinish, B.N. Singh, J. Nucl. Mater. 307\&311, 876 (2002)

9. H.L. Heinish, B.N. Singh, Philos. Mag. 83, 3661 (2003)

10. J.H. Evans, Philos. Mag. 85, 1177 (2005)

11. W. Frank, A. Seeger, R. Schindler, Radiat. Effects 40, 239 (1979)

12. A.H. Foster, J.M. Harder, D.J. Bacon, Mat. Sci. Forum 15-18, 849 (1987)

13. P.M. Derlet, D. Nguyen-Manh, S.L. Dudarev, Phys. Rev. B 76, 054107 (2007)

14. A.A. Semenov, C.H. Woo, Phys. Rev. B 74, 024108 (2006)

15. A.V. Barashev, S.I. Golubov, H. Trinkaus, Philos. Mag. 81, 2515 (2001)

16. A.D. Brailford, R. Bullough, J. Nucl. Mater. 69\&70, 434 (1978)

17. V.K. Sikka, J. Moteff, J. Nucl. Mater. 54, 325 (1974)

18. B.A. Loomis, S.B. Gerber, A. Taylor, J. Nucl. Mater. 68, 19 (1977)

19. B.A. Loomis, S.B. Gerber, J. Nucl. Mater. 102, 154 (1981)

20. K. Krishan, Radiat. Effects 66, 121 (1982)

21. C.H. Woo, W. Frank, J. Nucl. Mater. 140, 214 (1986)

22. A.A. Semenov, C.H. Woo, W. Frank, Philos. Mag. Lett. 85, 563 (2005)

23. J.H. Evans, Philos. Mag. Lett. 87, 575 (2007)

24. A.A. Semenov, C.H. Woo, E.A. Koptelov, Appl. Phys. A 73, 335 (2001)

25. A.A. Semenov, C.H. Woo, Phys. Rev. B 66, 024118 (2002)

26. A.M. Ovcharenko, C.H. Woo, A.A. Semenov, J. Nucl. Mater. 341, 201 (2005)

27. K.-Y. Liou, H.V. Smith Jr., P. Wilkes, G.L. Kulcinski, J. Nucl. Mater. 83, 335 (1979)

28. A.A. Semenov, C.H. Woo, J. Nucl. Mater. 233-237, 1045 (1996)

29. A.A. Semenov, C.H. Woo, Appl. Phys. A 69, 445 (1999)

30. C.H. Woo, B.N. Singh, Philos. Mag. A 65, 889 (1992)

31. D.J. Bacon, Yu.N. Osetsky, R. Stoller, R.E. Voskoboinikov, J. Nucl. Mater. 323, 152 (2003)

32. Y.N. Osetsky, D.J. Bacon, B.N. Singh, J. Nucl. Mater. 307\&311, 866 (2002)

33. R. Pasianot, M. Alurralde, A. Almazouzi, M. Victoria, Philos. Mag. 82, 1671 (2002)

34. J.F. Stubbins, J. Moteff, A. Taylor, J. Nucl. Mater. 101, 64 (1981)

35. G.L. Kulcinski, J.L. Brimhall, H.E. Kissinger, J. Nucl. Mater. 40, $166(1971)$

36. D.J. Mazey, S. Francis, J.A. Hudson, J. Nucl. Mater. 47, 137 (1973)

37. J.H. Evans, J. Nucl. Mater. 88, 31 (1980)

38. A. Almazouzi, M. Victoria, M.J. Caturla, T.D. de la Rubia, in EPFL supercomputing review, vol. 10. Swiss Fed. Inst. of Technology (1998)

39. K. Morishita, T.D. de la Rubia, J. Nucl. Mater. 271\&272, 35 (1999)

40. W. Horsthemke, R. Lefever, Noise-Induced Transitions (Springer, Berlin, 1984)

41. L.J. Chen, A.J. Ardell, J. Nucl. Mater. 75, 177 (1978) 